\title{
Revisión de evidencia del impacto de la anestesia obstétrica en los desenlaces maternos y neonatales. Un análisis de la literatura
}

\author{
Review of evidence of the impact of obstetric anesthesia on maternal \\ and neonatal outcomes. An analysis of the literature \\ Antonia Cárdenas ${ }^{1}$, Héctor J. Lacassie ${ }^{2 *}$ \\ Anestesióloga. \\ 2 Profesor Asociado en Anestesiología. \\ División de Anestesiología, Escuela de Medicina, Facultad de Medicina, Pontificia Universidad Católica de Chile.
}

Fuente de financiamiento: Fondos departamentales.

Conflicto de intereses: Los autores declaran no tener conflicto de intereses.

Fecha de recepción: 16 de julio de 2020 / Fecha de aceptación: 17 de julio de 2020

\begin{abstract}
We present the analysis and comments of a review of evidence of the impact of obstetric anesthesia on maternal and neonatal outcomes, based on an article previously published by Lim et al.[1]. The advances in obstetric anesthesia on analgesia and anesthesia for labor and delivery, anesthesia for cesarean section and outcomes in obstetric anesthesia.
\end{abstract}

Key words: Obstetric anesthesia, analgesia, pregnancy, labor.

\section{RESUMEN}

Se presenta el análisis y comentarios de una revisión de evidencia del impacto de la anestesia obstétrica en los desenlaces maternos y neonatales, basado en un artículo previamente publicado por Lim y cols.[1]. Se analizan los avances en la anestesiología obstétrica sobre analgesia y anestesia para el parto, anestesia para cesárea y desenlaces en anestesia obstétrica.

Palabras clave: Anestesia obstétrica, analgesia, embarazo, trabajo de parto.

a anestesia obstétrica actúa como puente entre disciplinas como la obstetricia, medicina materno-fetal, neonatología, cirugía general y anestesiología. Las investigaciones en esta área han evolucionado desde el uso de agentes volátiles para anestesia en el trabajo de parto, a los opioides y posteriormente, a las técnicas neuroaxiales.
La anestesia obstétrica nace en 1847 al introducirse el éter como analgesia para el trabajo de parto, técnica efectiva, pero con un perfil de seguridad desconocido para el feto y la madre. La analgesia del trabajo de parto fue evolucionando impulsada por las mismas pacientes, quienes la exigían, pasando por el uso de morfina y escopolamina, hasta la anestesia general para 
la cesárea, la cual se asoció a complicaciones en el manejo de la vía aérea y muertes maternas. A partir de la década de 1980 se masificó el uso de la analgesia neuroaxial en el parto, cuyo perfil de seguridad fue mejorado por avances como la dosis de prueba epidural, el uso de dosis epidurales incrementales, la eliminación de la bupivacaína 0,75\% y el tratamiento con lípidos para la intoxicación sistémica con anestésicos locales, entre otros.

Los anestesiólogos obstétricos han contribuido a distintas iniciativas a favor de la seguridad materna, no solo en la analgesia propiamente tal, habiendo demostrado que no influye en el riesgo de aumentar los partos por cesárea, sino que también mejorando las estrategias de manejo del dolor posparto, en estudios de comorbilidad materna, desenlaces neonatales, etc.

Esta revisión se centra en los avances de la anestesia obstétrica de las últimas dos décadas, con énfasis en la última. Resume en gran parte el artículo de Lim y cols.[1].

\section{Analgesia y anestesia para el parto}

\section{Modalidades de analgesia para el parto}

\section{Analgesia neuroaxial}

Inicio y mantención: Suele iniciarse con una técnica de analgesia epidural o combinada espinal-epidural (CSE). La CSE tiene una menor latencia (2-5 min) que la epidural (15-20 min), genera bloqueo sensitivo más uniforme y mejor cobertura de segmentos sacros. Si bien se temía no poder probar el adecuado funcionamiento del catéter epidural, se ha visto que con la técnica CSE suelen fallar menos, tanto en el parto como en las cesáreas. La CSE se asocia a un mayor riesgo de taquisistolía uterina, por disminución rápida de catecolaminas circulantes (efecto tocolítico) y un posible mayor riesgo de bradicardia fetal, transitoria, que no condiciona un aumento en las operaciones cesáreas de emergencia[2].

Existe una modificación de la técnica CSE: analgesia epidural con punción dural, que es una CSE, pero sin inyectar medicamentos intratecales. Al compararla con la analgesia epidural presenta mejor analgesia sacra y menos prurito, hipotensión arterial, taquisistolía y requerimiento de dosis suplementarias de analgésicos. Esto se explicaría por translocación de los medicamentos al espacio subaracnoideo[3].

Se recomienda iniciar y mantener la analgesia epidural con dosis bajas de anestésicos locales (AL) y opioides, de manera de minimizar el riesgo de: intoxicación sistémica por anestésicos locales (LAST), producir una anestesia espinal total y minimizar los efectos hemodinámicos y el paso trasplacentario de droga. Se suele iniciar la analgesia epidural con anestésicos locales tipo amino-amida de larga duración junto con un opioide (en general 5-15 ml de bupivacaína 0,0625\%-0,0125\% con 50-100 ug de fentanyl o 5-10 ug de sufentanil). La droga por usar al iniciar la CSE depende de la etapa del trabajo de parto. Al inicio de la primera etapa (fase latente) se puede utilizar una dosis intratecal de opioides (ej.: 20-25 ug de fentanyl) para el manejo del dolor visceral, mientras que en la fase activa de la primera etapa y en la segunda etapa se recomienda un anestésico local asociado a un opioide (bupivacaína 1-2,5 mg con 15-20 ug de fentanyl), de manera de cubrir el dolor somático también. Se suele mantener la analgesia epidural con una infusión continua de bupivacaína 0,0625\%-0,1\% con fentanyl 1,5-3 ug/ml a una velocidad de $8-15 \mathrm{ml} / \mathrm{h}$, ya sea sola o en el contexto de una analgesia controlada por la paciente.

\section{Infusión epidural continua vs bolo intermitente progra- mado}

El uso de infusiones epidurales continuas demostró un mejor manejo analgésico que el bolo manual intermitente, viéndose superado, posteriormente, por la analgesia controlada por el paciente (PCA por patient controlled analgesia) asociada a una infusión, la cual tiene un consumo total menor de anestésicos, menos incidencia de bloqueo motor y menor necesidad de intervenciones por el tratante.

Recientemente se ha investigado el uso de bolos epidurales intermitentes programados (PIEB por programmed intermittent epidural bolus), los cuales proporcionarían mejor analgesia por lograr una mejor distribución de la dosis en el espacio epidural. Comparado con la infusión continua ha demostrado mantener la analgesia con menor requerimiento de anestésicos, menos dolor incidental, menor riesgo de bloqueo motor y parto instrumental[4]. Una estrategia de programación es el uso de bupivacaína 0,0625\% con fentanyl $2 \mathrm{ug} / \mathrm{ml}$, administrándose un bolo de 6-8 $\mathrm{ml}$ cada 30-45 minutos y permitiéndose un bolo de PCA de 5-10 ml, con intervalo (lockout) de 10-15 minutos. Esta modalidad ha demostrado una muy buena satisfacción usuaria. Una desventaja potencial y teórica es el riesgo de presentar un bloqueo neurológico extenso en casos de migración del catéter al espacio intratecal (la administración en infusión es más segura en este aspecto que el bolo, y el bolo manual permite identificar la mal posición del catéter).

\section{Opioides sistémicos para analgesia del trabajo de parto}

Son de utilidad en los casos en que no se pueda utilizar analgesia neuroaxial (contraindicación o falla en lograr la punción). Puede utilizarse una PCA endovenosa de fentanyl, con 25 ug cada 10-15 min, con un lockout horario de 100 ug. Otra alternativa popular es el remifentanil, que es un opioide de ultracorta duración. Genera analgesia adecuada, aunque no es superior a la analgesia neuroaxial. Produce sedación materna y depresión respiratoria, por lo que requiere de monitorización materna continua. Se ha demostrado que el fentanyl produce menor depresión respiratoria materna que el remifentanil, pero mayor depresión neonatal.

\section{Óxido nitroso}

Es una técnica que ha demostrado satisfacción materna versus no usar analgesia, sin embargo, la satisfacción materna durante el trabajo de parto implica condiciones que van más allá de la analgesia, haciéndola un desenlace multidimensional. Es menos eficaz que la analgesia neuroaxial. Es seguro para las madres, pero falta evidencia de adecuada calidad sobre los desenlaces neonatales-infantiles y contaminación ambiental.

\section{Farmacogenómica}

Los avances en la medicina genética llevarán a un manejo personalizado del dolor en el futuro, sin embargo, si bien los factores genéticos pudieran explicar en parte la variabilidad interindividual en el dolor y progreso del trabajo de parto, el nivel de conocimiento actual en el tema aún es inadecuado para modificar el manejo de la analgesia del trabajo de parto. 


\section{Efecto de la analgesia en la progresión del trabajo de par- to y vía de parto}

\section{Analgesia neuroaxial y parto instrumental}

Hay una relación controversial entre la analgesia epidural y el parto instrumental. En la obstetricia moderna ha habido un descenso en la realización de partos instrumentales (fórceps), a favor de una mayor tasa de cesárea. Es fácil concluir que la analgesia del parto pudiera aumentar la tasa de cesáreas, sin embargo, lo más probable es que sea un confundente por indicación. Se ha visto mayor duración de la primera y segunda etapa del trabajo de parto (30 y 15 min) y más partos instrumentales al usar bupivacaína en altas dosis, en comparación con grupos de bajas dosis[5]. Además, muchos estudios han notado una relación entre la dosis total de anestésicos locales y bloqueo motor. Pero la asociación entre bloqueo motor y parto instrumental es inconsistente. La analgesia epidural actual busca minimizar el bloqueo motor con el uso de soluciones de anestésicos locales en bajas concentraciones. En una reciente actualización de los efectos de la analgesia neuroaxial, se vio que su uso no aumenta los partos instrumentalizados cuando se evaluaron los estudios realizados después del año 2005, posiblemente porque estos estudios incluyeron técnicas de anestesia obstétrica modernas, en que se utilizan bajas dosis de anestésicos[6].

\section{Vía de parto}

Las mujeres que requieren más analgesia neuroxial probablemente tienen un parto más doloroso. Los factores que se asocian al parto doloroso se pueden asociar a un riesgo aumentado de cesárea[7]. Por esto se pensaba que la analgesia aumentaba la tasa de cesáreas. Una revisión sistemática de 2018 no encontró una relación entre la analgesia epidural en trabajo de parto y el riesgo de cesárea. Además, múltiples ensayos clínicos aleatorizados han demostrado que no hay relación entre el uso de analgesia epidural en la fase latente de dilatación y el riesgo de cesárea. Tampoco existe esta asociación entre el uso de CSE en fase latente y cesárea.

\section{Progresión del parto}

Existían resultados conflictivos sobre si la analgesia neuroaxial prolongaba o acortaba el trabajo de parto, principalmente por fallas metodológicas ya que existen distintas definiciones de inicio del trabajo de parto en las revisiones y el examen cervical puede verse postergado cuando hay buena analgesia. Wong y cols.[8] demostraron una disminución en la duración de la primera etapa del trabajo de parto al administrar analgesia neuroaxial en etapa precoz del trabajo de parto (antes de los $4 \mathrm{~cm}$ ). Por otro lado, se ha visto que una analgesia efectiva se asocia a una prolongación de la segunda etapa (expulsivo) de $30 \mathrm{~min}$. Dado los posibles efectos adversos en cuanto a desenlaces fetales y maternos, es importante realizar más estudios sobre esta asociación.

\section{Anestesia neuroaxial para versión externa}

La versión externa es la rotación manual del feto en podálica, entre las 36-39 semanas de gestación, con el objetivo de intentar un parto vaginal y prevenir la cesárea. El uso de anestesia neuroaxial se asocia a una mayor tasa de éxito del procedimiento en $13 \%-50 \%$, la que no es dosis dependiente (no genera cambio en el nivel de relajación abdominal, como se creía previamente). Se solía temer que pudiera enmascarar los síntomas de rotura uterina y desprendimiento de placenta, sin embargo, no hay diferencia en la tasa de esos eventos entre anestesia neuroaxial y general. La anestesia neuroaxial tiene la ventaja de poder utilizarse en caso de requerir una cesárea y como desventaja que puede causar hipotensión arterial materna y generar retraso del alta de la paciente por bloqueo motor.

\section{Ingesta oral en el trabajo de parto}

Tradicionalmente, existe un riesgo de mortalidad materna asociado a la aspiración del contenido gástrico favorecido por el desplazamiento del esfínter esofágico inferior (EEI) al tórax, disminución en $50 \%$ de la presión del EEI en el embarazo, disminución de motilina y el uso de opioides que prolongan el vaciamiento gástrico. Para reducir la morbimortalidad existe un enfrentamiento dirigido, donde 1) se ha difundido la preferencia por el uso de anestesia neuroaxial; 2) hay restricción de ingesta oral en el parto; 3) favorecer el uso de antiácidos preanestésicos; 4) inducción de anestesia general en secuencia rápida; 5) entrenamiento de manejo de vía aérea dirigido a anestesistas y 6) mejoría en el uso de dispositivos de manejo de vía aérea difícil. Todo esto ha conducido a una disminución de morbimortalidad y a una tendencia actual a la liberalización de la ingesta alimentaria durante el trabajo de parto. Un metaanálisis muestra que no hay correlación entre la ingesta alimentaria, la vía de parto y bienestar neonatal en partos de bajo riesgo, pero falta información respecto al riesgo de aspiración materna. Las guías ASA permiten la ingesta de líquidos claros durante el trabajo de parto. La estrategia de ayuno para la cesárea electiva sugiere 2 horas de ayuno para líquidos claros y 6-8 h para comida, según el nivel de grasa. Probablemente el tema del ayuno en el trabajo de parto siga siendo controversial. Por ahora se recomienda evitar alimentos sólidos y particulados, permitiéndose los líquidos claros, entre los que se incluyen: agua, jaleas, té, café, bebidas azucaradas e incluso bebidas energéticas o concentrados proteicos, siempre que sean claros.

\section{Anestesia para cesárea}

\section{Avances en anestesia espinal para cesárea}

Se prefiere la técnica espinal en dosis única por su simplicidad, confiabilidad y calidad del bloqueo logrado. Cuando se usa fentanyl o morfina intratecal la dosis efectiva 95\% (DE95) de la bupivacaína hiperbárica es de 13 mg[9]. Al agregar un opioide liposoluble (ej.: fentanyl), potencia el efecto del anestésico local, disminuyéndose la dosis total requerida, pero produce mayor hipotensión arterial y náuseas. El agregar morfina aporta analgesia por hasta $36 \mathrm{~h}$. La epinefrina como coadyuvante (0,1-0,2 mg) aumenta en 15\% la duración del bloqueo, lo que es clínicamente poco relevante. Finalmente, la clonidina mejora la analgesia, reduce calofríos e hiperalgesia, pero produce hipotensión arterial y sedación.

\section{Conversión de analgesia epidural en anestesia para ce- sárea}

Se recomienda el uso de 15-20 ml lidocaína al 2\% asociada a epinefrina 1:200.000, a lo que se puede agregar bicarbonato 
de sodio 8,4\% para alcalinizar y disminuir la latencia de la mezcla. Es importante convertir exitosamente la anestesia epidural, ya que se considera un estándar de calidad en Estados Unidos de Norteamérica y la anestesia general se asocia a desenlaces negativos como dolor y sedación posoperatoria, awareness, hemorragia posparto, aspiración y dificultad en la intubación traqueal. Los factores de riesgo de falla de conversión son: la urgencia del procedimiento; necesidad de dosis de refuerzo por el catéter durante el trabajo de parto; inicio de la analgesia con técnica epidural en comparación con CSE y anestesiólogo general vs obstétrico.

\section{Hipotensión arterial intraoperatoria}

La anestesia espinal produce hipotensión arterial por disminución de la resistencia vascular sistémica, la cual se acompaña de un aumento del gasto cardíaco. El vasopresor ideal para mantener el flujo útero placentario ha sido sujeto de investigación por décadas. La efedrina se asocia a acidosis fetal por paso transplacentario y activación metabólica fetal. Con fenilefrina se han evidenciado mejores desenlaces neonatales (mejor $\mathrm{pH}$ de arteria umbilical y menor exceso de base), junto a una menor incidencia de náuseas y vómitos maternos. Destaca que produce bradicardia materna, asintomática. La infusión profiláctica de fenilefrina es más efectiva que el uso en bolos y se recomienda titular para obtener una presión arterial similar a la basal. La mayoría de los estudios son en cesáreas electivas y pareciera ser que en cesáreas por preeclampsia el desenlace neonatal no se ve influenciado por cual vasopresor se usa. Por otro lado, otros estudios sugieren que la norepinefrina se acerca más al vasopresor ideal, ya que aumenta la frecuencia cardíaca y el gasto cardíaco en comparación a fenilefrina y se ha visto que en la sangre de arteria umbilical hay menor nivel de catecolaminas, mayor $\mathrm{pH}$ y contenido de oxígeno, lo que indicaría mayor paso de oxígeno transplacentario. Ante un primer episodio de hipotensión arterial, 8 ug de norepinefrina equivaldrían a 100 ug de fenilefrina (relación de potencia de 1:13,1)[10]. Aún falta evidencia para designarlo como estándar de cuidado.

\section{Oxígeno suplementario}

No hay evidencia que muestre beneficio ni daño clínicamente evidente por su uso en cesárea electiva y en embarazos normales, por lo que no se recomendaría utilizarlo de rutina. Falta evidencia en cesáreas en pacientes con comorbilidades o en reanimación.

\section{Dolor poscesárea}

Es un dolor heterogéneo y lo ideal sería poder predecirlo para realizar un manejo individualizado. Pan y cols. realizaron un cuestionario de tres preguntas para identificar aquellas pacientes que tendrán más dolor post cesárea, que es el primer paso hacia la analgesia individualizada. Actualmente, el estándar de manejo en Estado Unidos de Norteamérica es la analgesia multimodal con morfina intratecal, antiinflamatorios no esteroidales (AINEs) y acetoaminofeno (paracetamol), buscando limitar el uso de opioides sistémicos. La morfina neuroaxial tiene efectos adversos dosis dependiente (> $100 \mathrm{ug}$ ) y puede producir prurito, náuseas, retención urinaria y depresión respiratoria. Los AINEs reducen en $50 \%$ el consumo de opioides y 30\% la aparición de efectos adversos de ellos. El ketorolaco puede usarse de forma segura en la lactancia, teniendo como contraindicaciones la enfermedad renal y el antecedente de bypass gástrico con $Y$ de Roux. Además, el acetoaminofeno tiene un efecto aditivo con los AINEs y disminuye en $20 \%$ el consumo de opioides.

La morfina neuroaxial requiere de monitorización en el posoperatorio, lo que ha sido revisado recientemente por la Sociedad de Anestesia Obstétrica y Perinatología (SOAP), recomendando controlar de acuerdo a la dosis intratecal empleada (Tabla 1)[11].

\begin{tabular}{|c|c|c|}
\hline Factores de riesgo & Dosis de morfina neuroaxial & $\begin{array}{l}\text { Recomendaciones de monitorización } \\
\text { respiratoria posoperatoria }\end{array}$ \\
\hline \multirow[t]{3}{*}{ Sin factores de riesgo (sana y normopeso) } & $\begin{array}{l}\leq 50 \text { ug IT } \\
\leq 1 \mathrm{mg} \text { epidural }\end{array}$ & Sin monitorización adicional a la estándar posoperatoria \\
\hline & $\begin{array}{l}50-150 \text { ug IT } \\
1-3 \text { mg epidural }\end{array}$ & Evaluar FR y sedación cada $3 \mathrm{~h}$ por $12 \mathrm{~h}$ \\
\hline & $\begin{array}{l}>150 \text { ug IT } \\
>3 \text { mg epidural }\end{array}$ & $\begin{array}{l}\text { Seguir guías ASA } \\
\text { Control FR y sedación horaria primeras } 12 \mathrm{~h} \text {, luego } \\
\text { cada } 2 \mathrm{~h} \text { hasta completar } 24 \mathrm{~h} \\
\text { Considerar medidas adicionales según juicio clínico** }\end{array}$ \\
\hline $\begin{array}{l}\text { Con factores de riesgo: } \\
\text { - Preoperatorios: IMC } \geq 40 \text {, SAHOS, uso crónico o abuso } \\
\text { de opioides, HTA, administración de MG } \\
\text { - Posoperatorios: AG, uso de otros opiodes, sedantes*, } \\
\text { administración de MG, desaturación de oxígeno en } \\
\text { recuperación }\end{array}$ & Cualquier dosis & $\begin{array}{l}\text { Seguir guías ASA } \\
\text { Control FR y sedación horaria primeras } 12 \mathrm{~h} \text {, luego } \\
\text { cada } 2 \mathrm{~h} \text { hasta completar } 24 \mathrm{~h} \\
\text { Considerar medidas adicionales según juicio clínico** }\end{array}$ \\
\hline
\end{tabular}

*Ejemplos de agentes sedantes: benzodiacepinas, antieméticos con efecto sedante, anestesia general; **Cambio de frecuencia de control FR o uso de oxímetro de pulso.

Abreviaciones: IT: intratecal; FR: frecuencia respiratoria; IMC: índice de masa corporal; SAHOS: síndrome de apnea hipopnea obstructiva del sueño; HTA: hipertensión arterial; MG: sulfato de magnesio; AG: anestesia general; ASA: Sociedad Americana de Anestesiología; h: horas. 


\section{Bloqueo de nervio periférico}

La morfina intratecal que, si bien se asocia a efectos adversos, es la droga que tiene mejor desempeño en términos de analgesia poscesárea. Se puede mejorar la analgesia posoperatoria con bloqueos como el bloqueo de plano del transverso abdominal (TAP block), bloqueo del cuadrado lumbar e infiltración de herida operatoria. El TAP block sirve para el manejo del dolor somático, pero no el visceral. Si bien no es superior a la morfina, sí es útil cuando esta no es parte del plan de manejo. El bloqueo del cuadrado lumbar tiene como beneficio que se ubica en un espacio continuo con el paravertebral, por lo que se administraría analgesia en la cadena simpática. Por esto ha demostrado ser superior a la anestesia espinal sola y con TAP block, pero aún no se ha comparado con la morfina intratecal. Finalmente, la infiltración de herida con anestésicos locales ha mostrado beneficios si la cirugía fue con anestesia general y no espinal. La instalación con un catéter e infusión es menos efectiva que el uso de morfina endovenosa y AINEs. En conclusión, el estándar de oro es el manejo multimodal (morfina, AINEs y paracetamol) y la analgesia suplementaria es útil en caso de dolor incidental o cuando no se puede cumplir éste (por ejemplo: anestesia general, AINEs contraindicados o no hay posibilidad de monitorización posoperatoria frecuente si se va a utilizar morfina neuroaxial).

\section{Desenlaces en anestesia obstétrica}

\section{Efecto de la analgesia del trabajo de parto en el feto}

Ocasionalmente se observa bradicardia fetal posterior al inicio de la analgesia neuroaxial. En un metaanálisis del año 2016 se vio que este fenómeno pudiera ser más frecuente al utilizar la técnica CSE, aunque no se asocia a peores desenlaces neonatales[2]. La fisiopatología propuesta es que al dar analgesia intratecal, disminuye la epinefrina en favor de la norepinefrina circulante, y como aquella tiene efecto beta-adrenérgico, se pierde la protección tocolítica y se produce taquisistolía. Esta bradicardia suele ser autolimitada y recuperarse con medidas estándar de reanimación y, en ocasiones, uso de tocolíticos. Su presencia no produce mayor incidencia de cirugía de emergencia.

\section{Lactancia}

Si la analgesia neuroaxial produce algún efecto en la lactancia materna exclusiva, es controversial, ya que hay estudios que muestran que no hay efecto, otros con efecto benéfico y algunos con efecto negativo. En cada uno de ellos hay muchas variables confundentes y se considera más importante para una lactancia exitosa la posibilidad de realizar apego, contacto piel a piel y la existencia de apoyo a la lactancia. El último estudio al respecto vio que la mantención de la analgesia epidural con hasta $2 \mathrm{ug} / \mathrm{ml}$ de fentanyl en la mezcla no impactó en la tasa de éxito de lactancia materna exclusiva a seis semanas del parto. Tampoco está muy claro el rol al comparar la anestesia general con la neuroaxial. Algunos estudios muestran menor éxito en la lactancia a largo plazo al haber recibido anestesia general y se cree que el manejo del dolor posoperatorio podría influir

\section{Fiebre $y$ estudio de sepsis en el neonato}

La analgesia neuroaxial se asocia a fiebre durante el trabajo de parto, la cual es de origen inflamatorio y no infeccioso[12]. Esta se le ha denominado "fiebre epidural". Se producen temperaturas mayores a $38^{\circ} \mathrm{C}$ por liberación de citokinas. Se ha visto que las mujeres con niveles aumentados de IL-6 al ingreso tienen mayor riesgo de desarrollarla. Tradicionalmente, se ha asociado la fiebre materna a peores desenlaces neonatales y se ha visto que si hay fiebre epidural, el recién nacido tiene mayor riesgo de estudio de sepsis, pero no es claro si afecta el desenlace a corto y mediano plazo. Es importante hacer la diferenciación diagnóstica, ya que la fiebre secundaria a corioamnionitis o funisitis (inflamación del cordón umbilical), se asocia a malos desenlaces neonatales[13],[14].

\section{Desenlaces neurocognitivos}

Algunos estudios observacionales mostraban una relación entre el trastorno de espectro autista y la anestesia intraparto, pero tenían muchos factores confundentes. Actualmente, no hay evidencia que apoye un cambio de régimen analgésico o anestésico. Es un asunto que debería investigarse a futuro.

\section{Depresión}

La analgesia del trabajo de parto podría asociarse a una reducción del riesgo de depresión posparto. Estos hallazgos se basan en que existe una relación ya establecida entre el dolor y la depresión en la población no obstétrica, ya que las vías neurológicas del dolor psicológico se superponen con las del dolor físico. En un estudio se observó menor incidencia de depresión posparto en mujeres que planearon usar analgesia epidural que aquellas que no deseaban usarla y finalmente la solicitaron. Existen múltiples confundentes en estos estudios por lo que faltan más investigaciones para determinar la verdadera relación entre estos factores.

\section{Contribución de la anestesia a la seguridad materna}

\section{Mortalidad por anestesia}

La mortalidad por anestesia ha disminuido significativamente (1 por millón de nacidos vivos en EE.UU.). Actualmente, se asocia más a las complicaciones de la anestesia neuroaxial (anestesia espinal total, falla de catéteres epidurales o catéteres intratecales no reconocidos) que a la anestesia general. En este aspecto hay un importante rol de los anestesiólogos en la prevención de la mortalidad materna, ya sea directa o indirecta.

\section{Hemorragia posparto}

Es la principal causa mundial de morbilidad, paro cardiorrespiratorio y muerte materna, donde la mayoría de estas muertes son prevenibles. Se ha visto una mejora en los desenlaces en hemorragia obstétrica por la existencia de protocolos de manejo.

La fisiología de una embarazada es distinta a la de una mujer no embarazada, pues la hemorragia obstétrica grave se asocia a hipofibrinogenemia temprana. Por lo tanto, es importante medir el fibrinógeno y, si es menor a $200 \mathrm{mg} / \mathrm{dl}[15]$, se debe realizar monitorización y tratamiento agresivo. La Asociación Americana de Anestesiólogos (ASA) recomienda tratamiento temprano de hipofibrinogenemia. Es importante realizar una estrategia de transfusión más restrictiva que liberal, ya que esta se asocia a menor riesgo de infección, eventos cardíacos y 
muerte. Sin embargo, no se debe sub-resucitar a la paciente.

El Colegio Americano de Obstetricia y Ginecología recomienda el uso de dispositivos como el Cell Saver en pacientes con anticuerpos y sin reserva de glóbulos rojos o aquellas que no acepten transfusiones. Recientemente, se ha demostrado que el uso de Cell Saver en obstetricia es una medida costo efectiva para casos de alto riesgo, y no se justificaría para casos rutinarios o en los cuales el riesgo de sangrado es bajo[16].

Se está generando interés con el uso de exámenes para guiar el manejo de la hemorragia obstétrica, como las pruebas viscoelásticas u otras pruebas de laboratorios. Sin embargo, aún no se ha estudiado si la transfusión guiada por exámenes de laboratorio mejora los desenlaces maternos.

El World Maternal Antifibrinolitic Trial (Woman Trial)[17] mostró que el uso de agentes antifibrinolíticos como el ácido tranexámico, produce una reducción significativa en las muertes por hemorragia $(1,5 \%$ vs $1,9 \%$; riesgo relativo 0,81 ; IC $95 \%, 0,65$ a 1,00; $p=0,045)$. La necesidad de laparotomía para controlar el sangrado también se redujo (riesgo relativo
0,64 ; IC 95\%, 0,49 a 0,85; $p=0,002$ ) y la mortalidad materna global se redujo en $31 \%$ si se administra en las primeras $3 \mathrm{~h}$ posparto. El beneficio fue independiente de la causa del sangrado. No afectó la incidencia de histerectomía y eventos tromboembólicos. Se recomienda administrarlo lo antes posible en una hemorragia posparto. No se ha demostrado beneficios al usarlo profilácticamente.

\section{Sistema de alerta temprana (Early Warning Systems)}

La detección tardía de morbilidad materna lleva a malos desenlaces, por lo que el grupo de "The Modified Early Obstetric Warning System" (sistema de aviso obstétrico precoz modificado) determinó un sistema de tamizaje de morbilidad en base a signos vitales: presión arterial, frecuencia cardíaca, frecuencia respiratoria, respuesta neurológica y débito urinario. Esta herramienta tiene $89 \%$ de sensibilidad, 79\% de especificidad y valor predictivo negativo de $98 \%$. Se recomienda la acción inmediata si se presentan alguno de los criterios de la Tabla 2.

Tabla 2. Signos de alarma maternos

\begin{tabular}{|c|c|}
\hline Signo o síntoma & Diagnósticos diferenciales \\
\hline HTA con cefalea persistente o disnea & $\begin{array}{l}\text { Crisis hipertensiva/PE y complicaciones } \\
\text { Falla cardíaca }\end{array}$ \\
\hline $\mathrm{PAS}<90 \mathrm{mmHo}>160 \mathrm{mmHg}$ & $\begin{array}{l}\text { Crisis hipertensiva/PE y complicaciones } \\
\text { Tromboembolismo venoso } \\
\text { Hemorragia } \\
\text { Sepsis }\end{array}$ \\
\hline $\mathrm{PAD}>100 \mathrm{mmHg}$ & $\begin{array}{l}\text { Crisis hipertensiva/PE y complicaciones } \\
\text { Falla cardíaca }\end{array}$ \\
\hline $\mathrm{FC}<50$ o $>120 \mathrm{lpm}$ & $\begin{array}{l}\text { Hemorragia } \\
\text { Crisis hipertensiva/PE y complicaciones } \\
\text { Tromboembolismo venoso } \\
\text { Falla cardíaca } \\
\text { Sepsis }\end{array}$ \\
\hline $\mathrm{FR}<10$ o $>30 \mathrm{rpm}$ & $\begin{array}{l}\text { Hemorragia } \\
\text { Crisis hipertensiva/PE y complicaciones } \\
\text { Tromboembolismo venoso } \\
\text { Falla cardíaca } \\
\text { Sepsis }\end{array}$ \\
\hline $\mathrm{SpO}_{2}<95 \%$ & $\begin{array}{l}\text { Hemorragia } \\
\text { Crisis hipertensiva/PE y complicaciones } \\
\text { Tromboembolismo venoso } \\
\text { Falla cardíaca } \\
\text { Sepsis }\end{array}$ \\
\hline Oliguria $<35 \mathrm{ml} / \mathrm{h}$ por $\geq 2 \mathrm{~h}$ & $\begin{array}{l}\text { Falla cardíaca } \\
\text { Hemorragia } \\
\text { Crisis hipertensiva/PE y complicaciones } \\
\text { Sepsis }\end{array}$ \\
\hline Agitación materna, confusión o falta de respuesta a estímulos & $\begin{array}{l}\text { Hemorragia } \\
\text { Crisis hipertensiva/PE y complicaciones } \\
\text { Tromboembolismo venoso } \\
\text { Falla cardíaca } \\
\text { Sepsis }\end{array}$ \\
\hline
\end{tabular}

Abreviaciones: HTA: hipertensión arterial; PE: preeclampsia; PAS: presión arterial sistólica; PAD: presión arterial diastólica; FC: frecuencia cardíaca; lpm: latidos por minuto; FR: frecuencia respiratoria; rpm: respiraciones por minuto; $\mathrm{SpO}_{2}$ : oximetría de pulso. Adaptado de: The Modified Early Obstetric Warning System. 


\section{Protocolos de oxitocina}

El manejo activo de la tercera etapa del trabajo de parto disminuye el riesgo de hemorragia obstétrica. Existen investigaciones que han demostrado la forma segura de administrar oxitocina para su manejo. Esto porque se puede asociar a efectos adversos que, si bien son infrecuentes, pueden ser muy graves: alteraciones de la conducción cardíaca, vasoespasmo coronario, hiponatremia grave y convulsiones. Se ha visto que no se necesitan grandes dosis para lograr el efecto clínico deseado. Se administra como infusión por su vida media corta (1-5 min): el ED90 para una cesárea electiva es 0,3 U/min (18 U/h) y para una cesárea luego de trabajo de parto es $44 \mathrm{U} / \mathrm{h}$ por desensibilización de sus receptores[18]. Sus efectos adversos más comunes son náuseas, vómitos y depresión del segmento ST en el electrocardiograma[19].

\section{Conclusiones}

Los avances en anestesia obstétrica han conducido a un mejor cuidado de las pacientes. Todavía se debe progresar en muchas áreas, como el posparto agudo y su influencia en el dolor crónico, la influencia del dolor del parto en depresión materna y fiebre epidural, entre otros. Las investigaciones en las características fisiológicas individuales del dolor van a permitir personalizar las intervenciones anestésicas obstétricas. Se requieren estudios comparativos de efectividad de técnicas analgésicas, manejo de hemorragia y el efecto de las distintas técnicas en los desenlaces de las pacientes para seguir avanzando en esta disciplina.

\section{Referencias}

1. Lim G, Facco FL, Nathan N, Waters JH, Wong CA, Eltzschig HK A Review of the Impact of Obstetric Anesthesia on Maternal and Neonatal Outcomes. Anesthesiology. 2018; 129 (1): 192-215.

2. Hattler J, Klimek M, Rossaint R, Heesen M. The Effect of Combined Spinal-Epidural Versus Epidural Analgesia in Laboring Women on Nonreassuring Fetal Heart Rate Tracings: Systematic Review and Meta-analysis. Anesth Analg. 2016; 123 (4): 955-64.

3. Chau A, Bibbo C, Huang C-C, Elterman KG, Cappiello EC, Robinson JN, et al. Dural Puncture Epidural Technique Improves Labor Analgesia Quality With Fewer Side Effects Compared With Epidural and Combined Spinal Epidural Techniques: A Randomized Clinical Trial. Anesth Analg. 2017; 124 (2): 560-9.

4. Capogna G, Camorcia M, Stirparo S, Farcomeni A. Programmed intermittent epidural bolus versus continuous epidural infusion for labor analgesia: the effects on maternal motor function and labor outcome. A randomized double-blind study in nulliparous women., Anesth Analg, 2011, p 826-31.

5. Effect of low-dose mobile versus traditional epidural techniques on mode of delivery: a randomised controlled trial. Lancet. 2001; 358 (9275): 19-23.
6. Anim-Somuah M, Smyth RM, Cyna AM, Cuthbert A. Epidural versus non-epidural or no analgesia for pain management in labour. Cochrane database of systematic reviews (Online). 2018; 5: CD000331.

7. Panni MK, Segal S. Local anesthetic requirements are greater in dystocia than in normal labor. 2003; 98 (4): 957-63.

8. Wong CA, Scavone BM, Peaceman AM, McCarthy RJ, Sullivan JT, Diaz NT, et al. The risk of cesarean delivery with neuraxial analgesia given early versus late in labor. N Engl J Med. 2005; 352 (7): 655-65.

9. Carvalho B, Durbin M, Drover DR, Cohen SE, Ginosar Y, Riley $E T$. The ED50 and ED95 of intrathecal isobaric bupivacaine with opioids for cesarean delivery, 2005, p 606-12.

10. Ngan Kee WD. A Random-allocation Graded Dose-Response Study of Norepinephrine and Phenylephrine for Treating Hypotension during Spinal Anesthesia for Cesarean Delivery. Anesthesiology. 2017; 127 (6): 934-41.

11. Bauchat JR, Weiniger CF, Sultan P, Habib AS, Ando K, Kowalczyk JJ, et al. Society for Obstetric Anesthesia and Perinatology Consensus Statement: Monitoring Recommendations for Prevention and Detection of Respiratory Depression Associated With Administration of Neuraxial Morphine for Cesarean Delivery Analgesia. Anesth Analg. 2019; 129 (2): 458-74.

12. Sharma SK, Rogers BB, Alexander JM, McIntire DD, Leveno KJ. A Randomized Trial of the Effects of Antibiotic Prophylaxis on Epidural-Related Fever in Labor. Anesth Analg. 2014; 118 (3): 604-10.

13. Sultan $P$, David AL, Fernando R, Ackland GL. Inflammation and Epidural-Related Maternal Fever. Anesth Analg. 2016; 122 (5): 1546-53.

14. Sultan $P$, Segal S. Epidural-Related Maternal Fever. Anesth Analg. 2020; 130 (2): 318-20

15. Charbit B, Mandelbrot L, Samain E, Baron G, Haddaoui B, Keita $\mathrm{H}$, et al. The decrease of fibrinogen is an early predictor of the severity of postpartum hemorrhage. J Thromb Haemost. 2007; 5 (2): 266-73.

16. Lim G, Melnyk V, Facco FL, Waters JH, Smith KJ. Cost-effectiveness Analysis of Intraoperative Cell Salvage for Obstetric Hemorrhage. 2018; 128 (2): 328-37.

17. Collaborators WT. Effect of early tranexamic acid administration on mortality, hysterectomy, and other morbidities in women with post-partum haemorrhage (WOMAN): an international, randomised, double-blind, placebo-controlled trial. Lancet. 2017; 389 (10084): 2105-16.

18. Lavoie A, McCarthy RJ, Wong CA. The ED90 of prophylactic oxytocin infusion after delivery of the placenta during cesarean delivery in laboring compared with nonlaboring women: an updown sequential allocation dose-response study. Anesth Analg. 2015; 121 (1): 159-64.

19. Svanström MC, Biber $B$, Hanes $M$, Johansson $G$, Näslund $U$, Bålfors EM. Signs of myocardial ischaemia after injection of oxytocin: a randomized double-blind comparison of oxytocin and methylergometrine during Caesarean section, British Journal of Anaesthesia, 2008, p 683-9. 